\begin{tabular}{|c|c|c|c|c|c|c|c|c|c|c|c|}
\hline \multirow[b]{2}{*}{$\begin{array}{l}\text { Bezeich- } \\
\text { nung }\end{array}$} & \multicolumn{7}{|c|}{ Fet $t$} & \multicolumn{4}{|c|}{ Fettsäuren } \\
\hline & $\begin{array}{c}\text { Schmelz- } \\
\text { punkt }\end{array}$ & $\begin{array}{l}\text { Erstar- } \\
\text { rungs- } \\
\text { ponkt }\end{array}$ & $\begin{array}{l}\text { He hner' } \\
\text { sche } \\
\text { Zabl }\end{array}$ & $\begin{array}{l}\text { Rei- } \\
\text { chert- } \\
\text { Meis } \\
\text { sehe } \\
\text { Zahl }\end{array}$ & $\begin{array}{c}\text { Versei- } \\
\text { fangs- } \\
\text { zahl }\end{array}$ & Jodzahl & $\begin{array}{l}\text { Re. } \\
\text { frak. } \\
\text { tion }\end{array}$ & 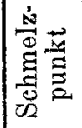 & $\begin{array}{l}\text { Erstar- } \\
\text { rungs- } \\
\text { punkt }\end{array}$ & $\begin{array}{l}\text { Versei- } \\
\text { fungs- } \\
\text { zahl }\end{array}$ & Jodzahl \\
\hline $\begin{array}{l}\text { Chinesi- } \\
\text { scherTalg }\end{array}$ & $\begin{array}{c}36,5 \text { bis } \\
44,5\end{array} \mid$ & $\begin{array}{c}26,7 \text { bis } \\
34\end{array}$ & $\cdots$ & - & $\begin{array}{c}179 \text { bis } \\
202\end{array}$ & $\begin{array}{c}28,1 \text { bis } \\
48,9\end{array}$ & - & $\begin{array}{c}39 \text { bis } \\
56,9\end{array}$ & $\begin{array}{c}34 \text { bis } \\
47,0\end{array}$ & $\begin{array}{c}181,2 \text { bis } \\
208,3\end{array}$ & $\begin{array}{c}30,31 \text { bis } \\
54,8\end{array}$ \\
\hline $\begin{array}{c}\text { Malabar- } \\
\text { Talg }\end{array}$ & $\left|\begin{array}{c}36,5 \text { bis } \\
37,5\end{array}\right|$ & 30,5 & $\begin{array}{l}95,14 \mathrm{bis} \\
95,21\end{array}$ & $\begin{array}{c}0,22 \text { bis } \\
0,44\end{array}$ & $\begin{array}{c}188,7 \text { bis } \\
191,9\end{array}$ & $\begin{array}{c}37,82 \mathrm{bis} \\
39,63\end{array}$ & 47,5 & 56,6 & 54,8 & - & - \\
\hline
\end{tabular}

Über das Vorkommen und Art dieser Fette gibt Benedikt-Ulzer folgendes an: "Chinesischer Talg wird aus den Früchten des chinesischen Talgbaumes, Stillingia sebifera, und verschiedenen Jatropha-Arten gewonnen. Die drei in einer Frucht enthaltenen Samen sind äußerlich mit einer harten, weißen Talgschicht bedeckt. Sie werden in große Holzzylinder gebracht und mit Wasserdampf behandelt, wobei der Talg abfliebt. Er kommt in harten, brüchigen, außen rötlich bestäubten, innen matt weißen Stücken in den Handel. In reinem Zustande macht er keine Fettflecken und ist geruch- und geschmacklos. Nach Maskelyne besteht der chinesische Talg ausschließlich aus Palmitin und Olein, und Hehner und Mitchell konnten in einer untersuchten Probe keine Stearinsäure finden. Der chinesische Talg findet in der Kerzen- und Seifenfabrikation dieselbe ausgedehnte Verwendung, wie auch der Malabartalg, dessen Fett aus den "Butterbobnen", den Samen von Vateria indica L. gewonnen wird."

Vielleicht geben diese Zeilen berufeneren Stellen Anlaß, der Verwendungsmöglichkeit derartiger Pflanzentalge zu Genußzzwecken nachzuforschen.

\title{
Über die Verwendbarkeit der „Vakuum-Destillation“ beim Ameisensäure-Nachweis.
}

Von

\section{Dr. Theodor Merl.}

Mitteilung aus der Kgl. Untersuchungsanstalt za Mänchen.

[Eingegangen am 20. März 1913.]

Seit einigen Jahren wird in physiologischen Laboratorien zur Bestimmung der flüchtigen Fettsäuren in tierisehen Flüssigkeiten oder Materialien ein Verfahren benutzt, bei welehem die Abscheidung der Säuren durch Destillation unter vermindertem Druck geschieht. Gegenüber den für diesen Zweck sonst üblichen Destillationsarbeiten soll durch das "Vakuumverfahren" vermieden werden, daß aus anderen im Untersuchungsmaterial mitvorhandenen organischen Stoffen durch Zersetzung Fettsäuren neu entstehen und zusammen mit der bereits ursprünglich enthaltenen Fettsäuremenge bestimmt werden, daß also bei der Analyse ein höherer Säuregehalt gefunden wird als dem gesuchten ursprünglichen entspricht. Es braucht hier nicht weiter erörtert zu werden, inwiefern auch für den Physiologen die möglichst genaue Feststellung von Fettsäuren 
nach Art und Menge, z. B. in den verschiedenen Ausscheidungsprodukten des tierischen Körpers, Wert und Interesse besitzt.

Steinkopf ${ }^{1}$ ) hat wohl zuerst darauf hingewiesen, daß man bei der gewöhnlichen Art der Vakuumdestillation statt des üblichen Luft- oder Gasstromes auch Wasserdampf zuleiten und so eine Wasserdamptdestillation im luftverdünnten Raume vornehmen kann. Dieser Anregung folgte E. Welde ${ }^{2}$, welcher das Verfahren zur Abscheidung bezw. quantitativen Bestimmung flüchtiger Fettsäuren benutzte. Während Welde sich darauf beschränkte, die Brauchbarkeit dieser Destillationsart zu prüfen und in verschiedenen Flüssigkeiten (Frauenmilch, Kuhmilch, Magen- und Darminhalt u. a.) die Gesamtmenge der enthaltenen flüchtigen Fettsäuren festzustellen, haben Edelstein und $\mathrm{Csonka}^{3}$ ) in den auf gleiche Weise erhaltenen Destillaten durch fraktionierte Fällung mit Silbernitrat auch die Art der Fettsäuren bestimmt; so fanden sie, daß bei ihrer Arbeitsweise die Essigsäure bis 94\%, Capron-, Capryl- und Buttersäure bis 98 und $100 \%$ bestimmt werden können. Es liegt dann noch eine neuere Studie von Strisowe $\mathbf{r}^{4}$ ) vor, welcher mittels Vakuumdestillation die in physiologischen und pathologischen Fällen im Harn vorkommende Ameisensäure abschied und in bekannter Weise bestimmte.

Anschliebend an diese Arbeiten haben auch die nachstehend beschriebenen Versuche Ameisensäurebestimmungen zum Gegenstand, welche unter Verwendung der Vakuumdestillation mit und ohne Wasserdampfzuleitung ausgeführt wurden. Meine Versuche beschäftigten sich speziell mit der Abscheidung der Ameisensäure aus sauren, zuckerhaltigen Flüssigkeiten, eine Frago, welche bekanntlich auch bei nahrungsmittelehemischen Untersuchungen eine Rolle spielt und erst durch die Arbeiten von Fincke befriedigend gelöst wurde.

In Ubereinstimmung mit der üblichen Auffassung, daß die Ameisensäure als ein häufig zu beobachtendes Oxydationsprodukt organischer Stoffe anzusehen sei, hält auch Strisower mit anderen Autoren diese Säure für ein gemeinsames, intermediäres Abbauprodukt der Kohlenhydrate, Fette und Eiweißkörper. Bezüglich der Zuckerarten findet diese Ansicht ohne weiteres eine Stütze in der vielfach gemachten Beobachtung, daß aus ihnen unter Säure- oder Alkalieinwirkung neben anderen Zersetzungsprodukten auch Ameisensüure in mehr oder minder großer Menge entstehen kann. Diese Tatsache wurde früher zu wenig berücksichtigt, sodaß schon Lieben ${ }^{5}$ ) darauf hinweisen konnte, daß alle älteren Angaben über das natürliche Vorkommen von Ameisensäure im Tier- und Pflanzenreiche mit Vorsicht aufzunehmen sind.

Vor allem für die genaue Feststellung kleiner und kleinster Ameisensäuremengen ist es selbstverständlich unerläßlich, die Entstehung von sekundärer Ameisensäure durch Zuckerzersetzung zu verhindern. Fincke ${ }^{6}$ ) hat gezeigt, in welcher Weise man diese Fehlerquelle vermeiden und die Neubildung von Ameisensäure wäbrend der Destillation in sauren zuckerhaltigen Flüssigkeiten auf ein praktisch zu vernachlässigendes Mindestmaß herabdrücken kann. Er stellte fest, daß selbst ganz schwach

1) Chem.Ztg. 1908, 32, 517.

2) Biochem. Zeitschr. 1910, 28, 504.

3) Biochem. Zeitschr. 1912, 42, 372.

4) Biochem. Zeitschr. 1913, 54, 189.

5) Monatshefte f. Chemie 1898, 19, 333.

6) Diese Zeitschrift 1911, 21, 1; 1912, 23, 255. - Biochem. Zeitschr. 1913, 51, 253-287. 
saure Invertzuckerlösungen bei der Wasserdampfdestillation eine Zersetzung erleiden: "Ameisensäurebildung ist dabei unvermeidlich." In solchen Fällen müsse man sich helfen durch nebenhergehende blinde Versuche mit Kontrollösungen oder durch Feststellung der Ameisensäure in gleich großen aufeinanderfolgenden Fraktionen des Destillates; in den nacheinanderfolgenden Fraktionen wird dann ohne Neubildung von Ameisensäure eine allmäbliche Abnahme, mit Neubildung ein Gleichbleiben oder eine Zunahme der Säure zu beobachten sein. Vollständig läBt sich also hier bei der gewöhnlichen Wasserdampfdestillation die Zuckerzersetzung und die Ameisensäureentstehung nicht vermeiden. So bemerkt auch $\mathrm{Kr}$ is ${ }^{1}$ ): „Es ist zurzeit nicht möglich auf qualitativem Wege zu entscheiden, ob einer sauren zuckerhaltigen Flüssigkeit Ameisensäure zugesetzt bezw. ob zur Herstellung eines Sirups ein ameisensäurehaltiger Fruchtsaft verwendet worden sei. Es kann dies nur durch eine Bestimmung der Ameisensäure geschehen, und auch dann hat man dem Umstande Rechnung zu tragen, daß Ameisensäure sich bei der Untersuchung bilden kann."

Die folgenden Versuche sollen nun zeigen, dal es doch möglich ist, aus sauren, stark zuckerhaltigen Flüssigkeiten darin enthaltene Ameisensäure abzuscheiden, obne daß während der Destillation eine Neubildung der Säure erfolgt. Es gelingt dies, wenn man unter Einhaltung ähnlicher Konzentrationsverhältnisse, wie sie Fincke angegeben, die Destillation unter vermindertem Druck ausführt. Das Verfahren der Destillation unter vermindertem Druck findet ja längst vielfache Anwendung und ist altbewährt überall da, wo man den Siedepunkt einer Substanz oder Lösung so weit herabdrücken will, daß Zersetzungen nicht mehr zu befürchten sind. Bei genügender Druckverminderung (20 mm Quecksilber und weniger) wird meist ein ganz erhebliches Sinken des Siedepunktes erreicht. So zeigt z. B. die reine Ameisensäure nach der Tabelle von Anschütz und Reitter ${ }^{2}$ ) folgende Zahlen:

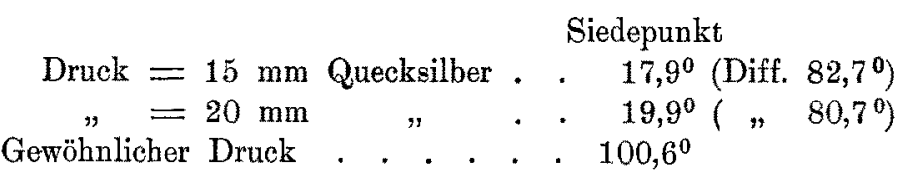

Damit ist also die Möglichkeit gegeben, selbst ziemlich konzentrierte Zuckerlösungen mit einem Siedepunkt von etwa $101^{\circ} \mathrm{C}$ (bei gewöhnlichem Druck) bei sehr mäbiger Siedetemperatur (etwa $40^{\circ} \mathrm{O}$ ) der Destillation zu unterwerfen und so den Zucker viel mehr zu schonen, als dies bei der gewöhnlichen Destillation, wo der Destillationskolben auf Asbest oder auf den Wasserbade erhitzt wird, möglich ist.

Für die Destillationen benutzte ich die gewöhnliche, von Anschütz und Reitter (1. c. S. 33) beschriebene Anordnung des Apparates. Die Vorrichtung ist in der von Welde etwas abgeänderten Form kurz folgende:

Für die Aufnahme des Untersuchungsmateriales dient ein Claisen-Kolben (Inbalt etwa $1100 \mathrm{ccm}$ ), dessen erste Öfnung eine durch Gummistopfen eingesetzte und an dem im Kolbeninneren mündenden Ende zur Capillare ausgezogene Glasröhre trägt, und auf dessen zweiter Öffnung ein mittels Gummistopfen aufgesetzter Glashahntrichter sitzt. Die seitliche Ansatzröhre des Destillationskolbens ist durch Gummi-

1) Mittlg. a. d. Gebiete d. Lebensm.-Untersuchung u. Hyg., veröffentl. v. Schweizer. Gesundh.-Amt 1912, 3, 210.

$\left.{ }^{2}\right)$ A sschüz und Reitter, „Die Destillation unter vermindertem Druck" im Laboratorium. 2. Aufl. Bonn 1895. 
stopfen mit einem geräumigen Vorlagekolben (Inhalt etwa $2000 \mathrm{cen}$ ) verbunden. Letzterer wird durch Klammer über einem größeren Trichter festgehalten, welcher das aus Glasrohr über den Kolben fließende Kühlwasser abführt, und steht durch ein Ansatzrohr mit einer kleinen eisgekühlten Saugflasche in Verbindung, welche zum Manometer und zur Saugpumpe führt. Durch die Glasröhre, die dem Destillationskolben aufsitzt, kann aus einem Dampfentwickler Wasserdampf oder nach Bedarf ein Luftstrom, welcher vorher durch zwei, mit Lauge bezw. konzentrierter Schwefelsäure gefullte Waschflaschen streichen muB, eingesaugt werden.

Das Arbeiten mit dieser Vorrichtung geht auch bei Wasserdampfuleitung glatt vor sich und bietet keine besonderen technischen Schwierigkeiten. Es wurde stets bei $10-15 \mathrm{~mm}$ Druck und einer Wasserbadtemperatur von etwa $60^{\circ} \mathrm{C}$ destilliert. Unter diesen Bedingungen war bei ungefähr $27 \%$-igen Zuckerlösungen die Innentemperatur bei der Destillation im Iuftstrom etwa $+35^{\circ} \mathrm{C}$ und im Wasserdampfstrom etwa $+43^{\circ} \mathrm{C}$.

Das in Mengen von je 100-120 ccm alle 10 Minuten nachzusaugende Wasser wurde vorher in einem entsprechend graduierten großen Becherglase, welches mit dem Glashahntrichter bezw. Destillationskolben durch heberartiges Glasrohr verbunden war, auf $45-60^{\circ} \mathrm{C}$ angewärmt. Bezüglich weiterer Einzelheiten verweise ich auf die Weld e'schen Ausführungen.

Die meisten Versuche wurden ausgeführt unter Benutzung sowohl des gewöhnlichen wie des Wasserdampf-Vakuumverfahrens. Die Flüchtigkeit der Ameisensäure mit Wasserdampf ist bekanntlich im allgemeinen eine geringere als die der folgenden Glieder der homologen Reihe der Paraffinmonocarbonsäuren (Fettsäurereihe). Die Gesetzmäfigkeiten, welche bei der Destillation verdünnter Lösungen solcher Säuren mit niedrigem Molekulargewicht und relativ bedeutender Löslichkeit in Wasser herrschen, hat A. Heiduschka') an dem Reichert-Meibl-Polenske'schen Destillationsverfahren experimentell geprüft und theoretisch begründet. $\mathrm{Er}$ fand hier, daß bei einem Gehalt der Lösung von 0,36 und $0,72 \%$ Ameisensäure etwa $40 \%$ der Ameisensäure übergehen. Bei der üblichen Wasserdampfdestillation gehen, wie Fincke zeigte, mit 1 Liter Destillat aus verschiedenen großen Flüssigkeitsmengen verschiedene Säuremengen über, wobei es weniger auf die absolute Menge der vorhandenen Ameisensäure ankommt. Nach Fincke gehen mit 1 Liter Destillat über

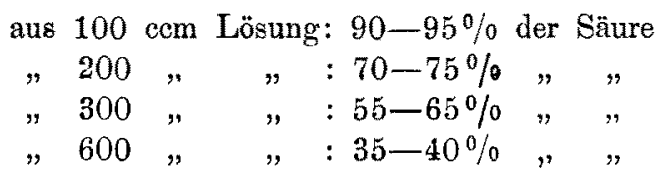

Bei der Destillation unter vermindertem Drucke gestalten sich nun die Ausbeuten noch günstiger, indem bei etwa 1 Liter Destillat aus $200 \mathrm{ccm} 90-95 \%$ der Säure übergetrieben, d. h. bei genügend langer Destillationsdauer quantitative Ausbeuten erhalten werden können. Allerdings ist darauf hinzuweisen, daß es sich bei meinen Versuchen um kleine und kleinste Mengen der Säure handelte. Die Beobachtungen Strisower's, welcher bei den Kontrollbestimmungen etwa $96 \%$ der angewandten Ameisensäure wiederfand, werden durch meine in Tabelle.I aufgeführten Versuchsergebnisse durchaus bestätigt.

1) A. Heiduschka, Beiträge zar Chemie und Analyse der Fette. Habilitationsschrift, München 1909 . 
Tabelle 1.

\begin{tabular}{|c|c|c|c|c|}
\hline \multirow[b]{2}{*}{$\begin{array}{l}\text { Zusammensetzung und Ameisensäuregehalt } \\
\text { der Kontroll-Lösungen }\end{array}$} & \multirow{2}{*}{$\begin{array}{c}\text { Mit } \\
\text { Wasser } \\
\text { aufgefüllt } \\
\text { auf } \\
\text { ccm }\end{array}$} & \multicolumn{3}{|c|}{ Ausbeute an Ameisensäure } \\
\hline & & $\begin{array}{l}\text { Wasser- } \\
\text { dampf- } \\
\text { Vakuum- } \\
\text { Verfahren } \\
g\end{array}$ & $\begin{array}{l}\text { Gewohn- } \\
\text { liches } \\
\text { Vakuum- } \\
\text { Verfahren } \\
\quad \text { g }\end{array}$ & $\begin{array}{l}\text { Ausbeute } \\
\text { in } \%\end{array}$ \\
\hline 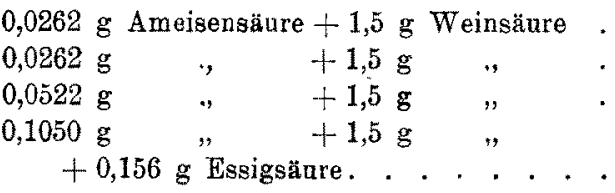 & $\begin{array}{l}300 \\
300 \\
300\end{array}$ & $\begin{array}{l}0,02499 \\
0,02643 \\
0,05109\end{array}$ & - & $\begin{array}{r}95,4 \\
100,8 \\
97,9 \\
100,3\end{array}$ \\
\hline 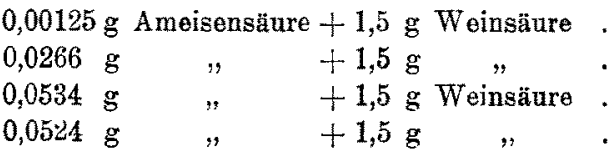 & $\begin{array}{l}250 \\
200 \\
300 \\
300\end{array}$ & - & $\begin{array}{l}0,00121 \\
0,02604 \\
0,05154 \\
0,05112\end{array}$ & $\begin{array}{l}96,8 \\
97,9 \\
96,5 \\
97,6\end{array}$ \\
\hline $\begin{array}{l}0,0262 \mathrm{~g} \text { Ameisensäure }+81 \mathrm{~g} \text { Saccharose }+ \\
1,5 \mathrm{~g} \text { Weinsäure } \\
0,0260 \mathrm{~g} \text { Ameisensäure }+81 \mathrm{~g} \text { Saccharose }+ \\
1,5 \mathrm{~g} \text { Weinsäure } \\
0,1040 \mathrm{~g} \text { Ameisensäure }+81 \mathrm{~g} \text { Saccharose }+ \\
1,5 \mathrm{~g} \text { Weinsäure } \\
\text {. . . . . . . . . . . }\end{array}$ & $\begin{array}{l}300 \\
300 \\
300\end{array}$ & $\begin{array}{l}0,0242 \\
0,0252 \\
0,1038\end{array}$ & - & $\begin{array}{l}92,4 \\
96,9 \\
99,8\end{array}$ \\
\hline $\begin{array}{l}0,0524 \mathrm{~g} \text { Ameisensäure }+81 \mathrm{~g} \text { Saccharose }+ \\
1,5 \mathrm{~g} \text { Weinsäure } \\
0,0529 \mathrm{~g} \text { Ameisensäure }+81 \mathrm{~g} \text { Glykose (Kahl- } \\
\text { ba u m) }+1,5 \mathrm{~g} \text { Weinsäure . . . . }\end{array}$ & 360 & - & 0,0520 & 100,5 \\
\hline
\end{tabular}

Aus den gefundenen Werten erhellt, daß kleine und kleinste Ameisensäuremengen sowohl aus rein wässerigen, weinsauren Lösungen, als auch bei Gegenwart von Kohlenhydraten unter vermindertem Druck abdestilliert werden können. Es war nun die weitere Frage zu prüfen, ob unter den gleichen Versuchsbedingungen eine Neubildung von Ameisensäure durch Zuckerzersetzung eintritt. Die hierzu nötigen Destillationen wurden absichtlich mit großen Mengen der bäufigeren Zuckerarten durchgeführt, damit eine etwaige Entstehung von Amessensäure durch Zersetzung der Kohlenhydrate besonders auffällig in die Erscheinung treten sollte. Für einen Teil dieser Versuche mußte eine möglichst ameisensäurefreie Invertzuckerlösung hergestellt werden. Zur Invertase-Inversion der Saccharose wurde als Hydrolysator stärkefreie Preßhefe benutzt (Thompson'sches Verfahren ${ }^{1}$ )). Erfahrungsgemäß besitzt das Ferment, so lange es sich noch in der Hefenzelle befindet, größere Widerstandsfähigkeit als die abgeschiedene mehr oder minder reine Invertase. Während die verzchiedenen Hefenarten bei böherer Temperatur (nach Hansen bei $52-62^{\circ}$ ) ihr Gärungsvermögen verlieren und bei $60-70^{\circ}$ absterben ${ }^{2}$ ), wird ihre Hydrolysierungsfähigkeit in Saccharoselösungen von $20-40 \%$ nach $\mathrm{Kjeldahl}$ bei $65^{\circ}$ erst geschwächt ${ }^{3}$ ). Um also bei Gewinnung der Invertzuckerlösung den Eintritt der Gärung zu vermeiden, wurde nach Thompson in folgender Weise verfahren:

1) Journal Chemical Society London $59,46$.

2) Lippmann, Chemie der Zuckerarten 1, 386.

$\left.{ }^{3}\right)$ Daselbst 2, 1297. 
$257 \mathrm{~g}$ ungebläuter Frankenthaler Würfelzucker wurden in etwa $700 \mathrm{ccm}$ Wasser gelöst. Dann wurden $27 \mathrm{~g}$ stärkefreie Preßhefe mit einem Teile der auf $55^{\circ} \mathrm{C}$ erwärmten Zuckerlösung angerieben und mit der Hauptmenge der Zuckerlösung in einem Erlenmeyer-Kolben vereinigt. Der Kolbeninhalt wurde unter zeitweiligem Rühren mit dem Thermometer 4 Stunden lang auf einer Temperatur von $55^{\circ} \mathrm{C}$ (Wasserbad) gehalten, sodann aufgekocht und nach dem Abkühlen bei $15^{\circ} \mathrm{C}$ auf $1000 \mathrm{ccm}$ gebracht und filtriert. Aus dem Filtrat, welches 25,72\% Invertzucker (nach Allihn) enthielt (der größte Teil der Saccharose war also invertiert), wurde durch Bleiacetat-Natriumsulfatfällung die Hauptmenge der gelösten Hefenproteine herausgeschafft. Die wasserklare, völlig farblose Invertzuckerlösung war nunmehr für meine Zwecke brauchbar.

Die bei den Versuchen erhaltenen Destillate (je etwa $1200 \mathrm{ccm}$ ) wurden durch Zusatz von $1 / 4$ N.- oder $1 / 1$ N.-Lauge oder durch Kochen mit gefälltem Calciumcarbonat am Rückflußkühler neutralisiert, dann zuerst auf dem Drahtnetz, schließlich auf dem Wasserbade in Porzellanschalen eingedampft. Der Rückstand wurde nach dem Quecksilberchlorid-Verfahren in bekannter Weise geprüft. Der Nachweis von Ameisensäure galt für erbracht, wenn aufer dem Eintritt einer durch Ammoniak sich schwärzenden weißen Fällung in einer Parallelprobe auch die Reaktion nach Fenton und Sisson in der von $\mathrm{F}_{\text {in }} \mathrm{ke}^{1}$ ) angegebenen Arbeitsweise positiv erhalten wurde.

Tabelle II enthält die mit Zuckerarten unter verschiedenen Versuchsbedingungen gewonnenen Ergebnisse.

Tabelle. II.

\begin{tabular}{|c|c|c|}
\hline \multirow{2}{*}{$\begin{array}{l}\text { Zusammensetzung der Lösungen } \\
\text { (mit Wasser auf } 300 \mathrm{ccm} \text { gefüllt) }\end{array}$} & \multicolumn{2}{|c|}{ Ameisensäure im Destillat } \\
\hline & $\begin{array}{l}\text { Wasserda mpf. } \\
\text { Vakuum-Verfabren }\end{array}$ & $\begin{array}{l}\text { Gew öhnliches } \\
\text { Vakuum.Verfahren }\end{array}$ \\
\hline $81 \mathrm{~g}$ Saccharose $+1,5 \mathrm{~g}$ Weinsäure & $\begin{array}{c}\text { Destillat } \quad \text { I: } 0,000517 \\
, \quad \text { II }: 0,000351\end{array}$ & $\begin{array}{c}\text { Destillat I: } 0,000341 \\
, \text { II: }\end{array}$ \\
\hline 81 g Dextrose („Kahlbaum“) $+1,5 \mathrm{~g}$ Weinsäure & $\begin{array}{c}\text { Destillat } \quad \text { I: } 0,00214 \\
" \quad \text { II: } 0,00069 \\
" \quad \text { III: } 0,00112\end{array}$ & $\begin{array}{c}\text { Destillat I: } \\
\Rightarrow \quad \text { II: } \\
\Rightarrow \text { III: }\end{array} \begin{array}{c}\text { keine } \\
\text { Aneisen } \\
\text { säure }\end{array}$ \\
\hline 81 g Lävulose (käufl., „Kahlbaum“) & $\begin{array}{c}\text { Destillat I: } 0,00201 \\
" \quad \text { II: } 0,00306\end{array}$ & - \\
\hline $\begin{array}{l}81 \text { g Lävulose (känfl., "Kahlbaum“) } \\
+1,5 \text { g Weinsäure }\end{array}$ & $\begin{array}{c}\text { Destillat } \text { I: } 0,0581 \\
, \quad \text { II }: 0,0134\end{array}$ & - \\
\hline $81 \mathrm{~g}$ Lävulose (krystallisiert, „Kahlbaum & $\begin{array}{c}\text { Destillat I: } 0,00087 \\
. \quad \text { II: } 0,00102\end{array}$ & - \\
\hline $\begin{array}{c}81 \text { g Lävulose (krystallisiert, „Kahlbaum*) } \\
+1,5 \mathrm{~g} \text { Weinsäure }\end{array}$ & $\begin{array}{cc}\text { Destillat } & \text { I: } 0,00638 \\
" & \text { II: } 0,00126 \\
" & \text { III: } 0,00149 \\
" & \text { IV: } 0,00104\end{array}$ & 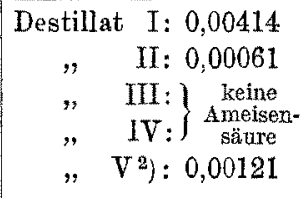 \\
\hline $77,16 \mathrm{~g}$ Inverìzucker $+6,0 \mathrm{~g}$ Saccharos $\theta$ & Keine Ameisensäure & Keine Ameisensäure \\
\hline
\end{tabular}

1) Diese Zeitschrift $1913, \mathbf{2 5}, 389$.

2) Bei Wasserdampfauleitung. 


\begin{tabular}{|c|c|c|}
\hline \multirow{2}{*}{$\begin{array}{l}\text { Zusammensetzung der Lösungen } \\
\text { (mit Wasser auf } 300 \mathrm{ccm} \text { gefüllt) }\end{array}$} & \multicolumn{2}{|c|}{ Ameisensäure im Destillat } \\
\hline & $\begin{array}{l}\text { Wasserdampf- } \\
\text { Vakuum-Verfahren }\end{array}$ & $\begin{array}{l}\text { Gewöhnliches } \\
\text { Vakuum-Verfabren }\end{array}$ \\
\hline $\begin{array}{c}\text { 77,16 g Invertzucker }+6,0 \text { Saccharose } \\
+1,5 \mathrm{~g} \text { Weinsäure }\end{array}$ & $\begin{array}{c}\text { Destillat I: } 0,00175 \\
\text { " II: } 0,00103\end{array}$ & $\begin{array}{c}\text { Destillat I: } 0,00164 \\
\Rightarrow \quad \text { II: } 0,00032 \\
" \quad \text { III : keine } \\
\text { Ameisensäure } \\
\text { (III: naeh 48-stündigem } \\
\text { stehen dex Löstugg) }\end{array}$ \\
\hline $81 \mathrm{~g}$ Saceharose $+3 \mathrm{~g}$ Weinsäare & Destillat: 0,000556 & $\begin{array}{l}\text { Destillat I: } 0,00041 \\
\quad " \quad \text { II: Keine } \\
\text { Ameisensäure }\end{array}$ \\
\hline 81 g Saccharose $+0,65 \%$ Phosphorsäure & Keine Ameisensäure & Keine Ameisensäure \\
\hline 81 g Saccharose $+1,5 \%$ Phosphorsäure & Destillat: 0,00056 & Keìne Ameisensäure \\
\hline $\begin{array}{c}81 \mathrm{~g} \text { Saccharose }+1,5 \mathrm{~g} \text { Weinsäure } \\
+3 \mathrm{~g} \text { Tannin }\end{array}$ & - & Keine Ameisensäure \\
\hline
\end{tabular}

Aus den in vorstehender Tabelle aufgeführten Zahlen und Beobachtungen ist ersichtlich, daß auch bei der Vakuumdestillation unter Wasserdampfzuleitung geringe Neubildung von Ameisensäure auftritt, nicht dagegen bei der gewöhnlichen Vakuumdestillation. Überall da, wo bei letzterem Verfahren anfänglich Ameisensäure nachgewiesen wurde, rührte sie nicht von Zersetzung, sondern von nicht völlig reinem Zucker her. Dies geht daraus hervor, daß nach dem Abdestillieren des geringen, als Verunreinigung vorhandenen Ameisenzäuregehaltes bei weiterem Destillieren neue Mengen davon nicht mehr nachzuweisen waren. Andererseits tritt auch in den Fällen, wo von vorneherein Ameisensäure nicht nachzuweisen ist, diese Säure sofort auf, sobald mit der nämlichen Lösung ein weiteres Destillat- - jetzt aber unter Wasserdampfzuleitung - gewonnen wird (vergl. die Versuche mit Dextrose und Lärulose). Somit ist erwiesen, daß unter den mehrfach erwähnten Voraussetzungen bei der Destillation unter vermindertem Druck, welche in der üblichen Weise unter Zuführen eines Luftstromes vorgenommen wird, eine Neubildung von Ameisensäure durch Zuckerzersetzung nicht eintritt.

In einer größeren Anzahl unzweifelhaft echter Bienenhonige von verschiedener Art und Herkunft, welche mir der Kgl. Bayerische Landesinspektor für Bienenzucht, Herr Carl Hofmann, verschafft hatte, war vor etwa 1 Jahr der Ameisensäuregehalt nach dem Fincke'schen Verfahren bestimmt worden. Da von einigen dieser Sorten noch genügend Material zur Verfügung stand, so war es von Interesse, mit diesem vergleichende Bestimmungen nach dem Vakuumverfahren auszuführen.

In Tabelle III sind die sämtlichen gefundenen Werte angegeben: 
Tabelle III.

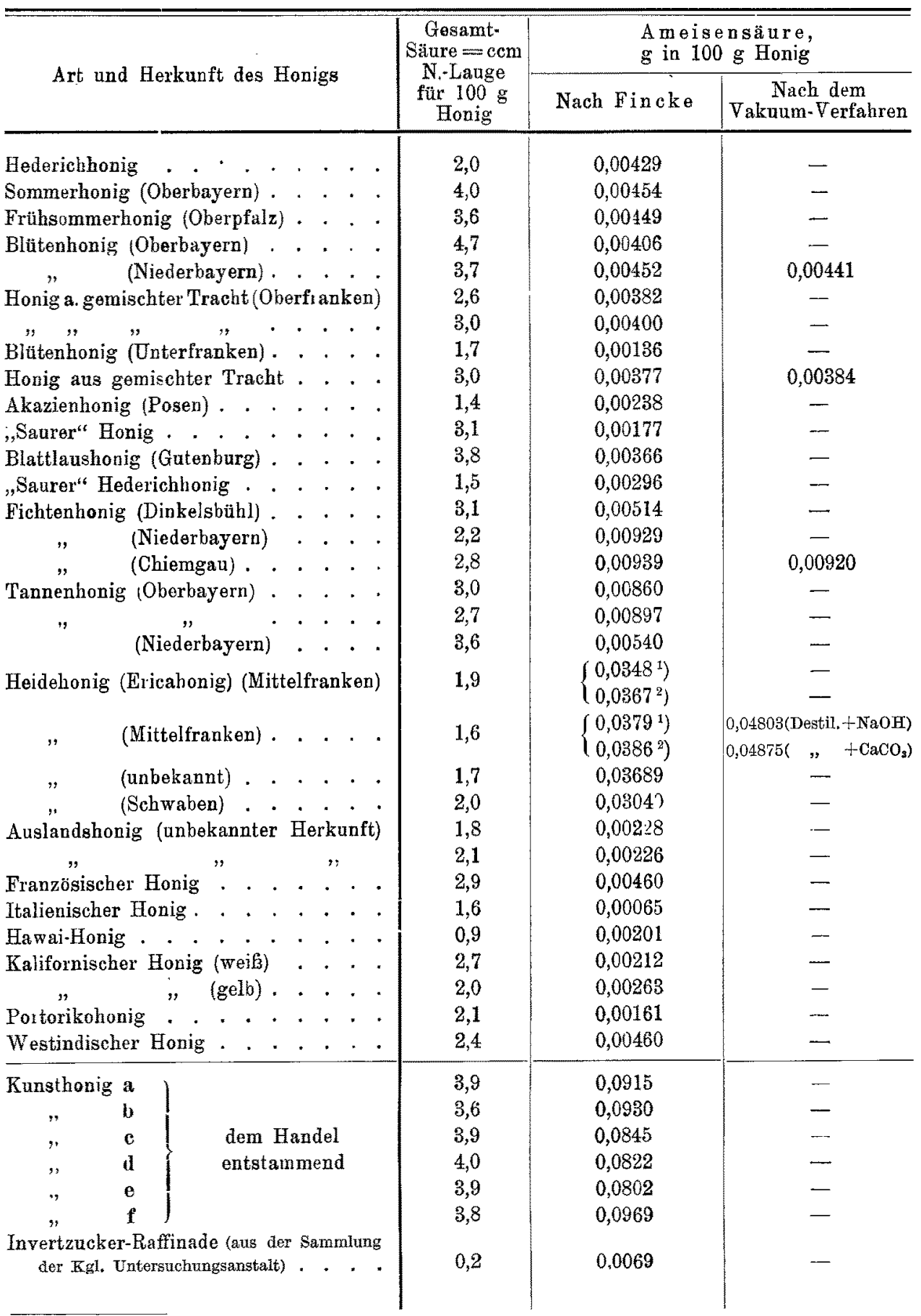

1) Als $\mathrm{HgCl}$ bestimmt.

2) Als CO bestimmt. 
In dem Heidehonig wurde nach dem Vakuum-Verfahren eine höhere Ameisensäurezahl gefunden wie nach Fincke. Diese auffallende Tatsache dürfte in dem Umstand ihre Erklärung finden, daß dieser Honig infolge hohen Eiweilgehaltes (Tanninfällung nach Lund: 4,7 ccm) stark schäumte, die Fincke'sche Durchdestillation deshalb sehr vorsichtig ausgeführt werden mufte und so vielleicht nicht der gesamte Ameisensäuregehalt übergetrieben wurde. Unter vermindertem Druck ging die Destillation glatter vor sich. Allerdings wurde letztere Bestimmung erst nach Jahresfrist ausgeführt. Eine Vermehrung des Ameisensäuregehaltes während dieser Zeit infolge Zuckerzersetzung ist zwar nicht ausgeschlossen, wird aber zunächst wohl nicht anzunehmen sein. Im übrigen werde ich mit Heidehonigen weitere Bestimmungen und Beobachtungen vornehmen. Die für die drei anderen (nach beiden Verfahren untersuchten) Honige gefundenen Zahlen stimmen sehr gut überein.

Diese paar Beispiele geben eine weitere Bestätigung der von Fincke experimentell bewiesenen Brauchbarkeit seines Verfahrens der Durchdestillation für die Honiguntersuchung. Ohne besondere Veranlassung wird man also bei Honigen das Vakuumverfahren nicht heranziehen, welches zwar die Zuckerzersetzung ganz ausschließt, immerhin aber umständlicher ist und weniger rasch zum Ziele führt.

Es können aber spezielle Fragen vorliegen, bei welchen man allein von diesem Verfabren eindeutige und entseheidende Ergebnisse erwarten kann. Ein solcher Fall, welcher zu dem Vorkommen von Ameisensäure im Bienenhonig in Beziehung steht, sei in folgendem geschildert:

Wenn man den Kopf einer Biene auf blaues Lackmnspapier legt und etwas quetscht, so ist zu beobachten, daß zwischen den Kiefern stark sauer reagierender Speichel herausgedrückt wird. Diese Erscheinung ist längst bekannt; ebenso die Tatsache, daß in Kopf und Thorax der Biene verschiedene Drüsen enthalten sind, von welchen die einen ein saueres, die anderen ein alkalischs Sekret absondern ${ }^{1}$ ).

Ferner wurde stets angenommen, daß in der Giftblase bezw. in dem Stachelapparat der Biene neben dem eigentlichen Gift auch Ameisensäure enthalten ist ${ }^{2}$ ).

Es ist nun eine alte umstrittene Frage, auf welche Ursachen das Vorkommen von Ameisensäure im Bienenhonig zurückzuführen sei. Rührt sie vom Stachelapparat her (M üll e nh of f) oder aus den Kopf- und Thoraxspeicheldrüsen (v on Plan ta $\left.{ }^{3}\right)$ ) oder ist sie lediglich aus Zucker durch Oxydation auberhalb des Bienenkörpers entstanden (Reidenbach ${ }^{4}$ ? ? 0 n Planta suchte die Säure der Kopf- und Thoraxspeicheldrüse durch Destillation abzuscheiden und ihre Art festzustellen; aus der Fähigkeit des Destillates, Silbernitrat zu reduzieren, schloß er auf die Anwesenheit von Ameisensäure. Reidenbach bemerkt mit Recht, daß diese Untersuchung ungenügend war und das Vorliegen von Ameisensäure nicht eindeutig bewiesen hat. Gegenüber der Müllenhoff'schen Ansicht behauptet Reidenbach, daß das Bienengift kaum merkliche Spuren der Säure enthalte. Es ist klar, daß, falls überhaupt diese Säure im Bienenkörper vorkommt, die Mengen nur außerordentlich geringe sein können und die Untersuchung, da mit der Anwesenheit von Zucker zu rechnen ist, auf die Mög-

1) Douhoff, Eichstadter Bienenztg. 1854-56. - P. Schiemenz, Über das Herkommen des Futtersaftes und die Speicheldrüsen der Biene etc.

$\left.{ }^{2}\right)$ Vergl. J. Langer, Chem.-Ztg. 1897, 21, 843.

3) Schweizer. Bienenztg. 1893, No, 5, 6 n. 10.

4) Pfälzer Bienenztg. 1911, No, 2-6. 
lichkeit der Neubildung von Ameisensäure während der Destillation Rücksicht nehmen muls.

400 Exemplare von Apis mellifica, welche durch Kälte getötet waren, wurden je in zwei Teile (Kopf und Thorax einerseits, Hinterleib anderseits) zerschnitten; die vereinigte Menge gleichartiger Teile (Kopf und Thorax mit den Speicheldrüsen Hinterleib mit Stachelapparat und der im Winter stark gefüllten Kotblase) wurde jede für sich, jedoch in genau gleicher Weise auf etwaigen Ameisensäuregehalt geprüft. Zu diesem Zwecke wurden die Teile in einer Reibschale zunächst für sich zerdrückt, dann mit destilliertem Wasser fein zerrieben und die Verreibung am Rückflußkühler auf $73^{\circ} \mathrm{C}$ (zur Eiweißgerinnung) erwärmt. Nach dem Erkalten wurde mit $1,0 \mathrm{~g}$ Weinsäure versetzt und die saure Mischung, ohne zu filtrieren, unter einem Drucke von $10-15 \mathrm{~mm}$ Quecksilber aus einem $60^{\circ} \mathrm{C}$ warmen Wasserbade in üblicher Weise destilliert. Die beiden Destillate von je etwa $1500 \mathrm{ccm}$ wurden mit Calciumcarbonat-Brei versetzt und einige Zeit am Rückflußkühler zum Kochen erhitzt. In dem eingedampften Filtrat wurde mittels Qecksilberchlorids der Ameisensäurenachweis zu führen versucht. In beiden Fällen hatte die Untersuchung ein negatives Ergebnis, d. h. weder im Kopf und Thorax noch im Hinterleib konnte Ameisensäure anch nur in Spuren nachgewiesen werden.

Es ist dies interessant, weil es beweist, daß der Bienenkörper - wenigstens in der Winterperiode - nicht die Quelle für den Ameisensäuregehalt des Honigs darstellen kann. Freilich wäre auch denkbar, daß gewisse Sekretionszellen der Drüsen erst während der Tracht, sozusagen unter der Reizwirkung der von der Natur reichlich dargebotenen Nektarschätze, diese Säure hervorzubringen vermögen, während diese ihre Funktion im Winter ruht. Zur gegebenen Zeit werde ich auch die hier nötige ergänzende Untersuchung nachholen.

Vielmehr Wahrscheinlichkeit hat aber die Reidenbach'sche Ansicht, dessen Versuche über die Oxydation des Zuckers an der Hand des Vakuumverfahrens gelegentlich nachgeprüt werden sollen. Bei der spontanen Oxydation des Zuckers ist Alkali nötig, wie neben anderen auch $M$ athews ${ }^{1}$ ) gezeigt hat; vielleicht spielen hierbei die ein alkalisehes Sekret liefernden Drüsen des Bienenkörpers eine Rolle, die bisher übersehen wurde.

Ich möchte an dieser Stelle dem Kgl. Landesinspektor für Bienenzucht, Herrn Carl Hofmann, für sein liebenswürdiges Entgegenkommen bei Beschaffung des für die Untersuchungen wertvollen Honig- und Bienenmaterials meinen besten Dank aussprechen.

Schließlich sei noch kurz über einige Vorversuche berichtet, welche über die Flüchtigkeit der Benzoesäure, Salicylsäure und Zim tsäu re bei der Vakuumdestillation angestellt wurden. Die Flüchtigkeit der genannten Säuren mit Wasserdämpfen wurde schon öfter $\mathrm{zu}$ ihrer Abscheidung benutzt ${ }^{2}$ ), und ich pflichte der Ansicht Lehmann's bei, welcher an Stelle des Ausschüttelns — namentlich bei zuckerreichem Material - es für praktischer hält, von Anfang an mit Wasserdämpfen zu destillieren. Da im Gegensatz zu der lästigen Emulsionsbildung und Lösung anderer störender

1) Journal of Biolog. Chemistry 1909, 6, 3-20; diese Zeitschrift 1911, 22, 517.

2) Vergl. Lehmann in Chem.-Ztg. 1908, 32, 949; von der Heide in dieser Zeitschrift 1910, 19, 138; C. Griebel in dieser Zeitschrift 1910, 19, 241; A. Kr $1918,26,12$. 
Stoffe durch das Extraktionsmittel bei der Destillationsarbeit die betreffende Säure in reinerer Form erhalten wird, geben die darauf gegründeten Verfahren in manchen Fällen besondere Vorteile. Auch mittels des Vakuumverfahrens gelingt es nach meinen bisherigen Erfahungen noch bei einem Gebalt von $0,25-0,5-1,0 \mathrm{mg}$ der genannten Säuren in $100 \mathrm{ccm}$ Flüssigkeit, im Destillat die Säuren einwandfrei und sicher nachweisen.

Ob hier quantitative Ausbeuten erhalten werden können, soll durch weitere Studien geprüft werden.

\section{Berichtigung.}

In meiner Arbeit: „Beiträge zur Beurteilung des Schweineschmalzes" (Diese Zeitschrift 1914, 27, 142) sind von mir in der Tabelle II die Schmelz- und Erstarrungspunkte sowie die Differenzzahlen der Proben No. 56 und 57 eine Reihe zu hoch gesetzt. Die Zahlen $45,3,24,47$ und 20,83 bei Probe No. 55 (S. 149) gehören zur Probe No. 56 und die Zablen 46,1,29,0 und 17,1 bei Probe No. 56 (S. 150) zu Probe No. 57.

Die Bestimmung der Schmelz- und Erstarrungspunkte bei den Proben No. 56 und 57 bezweckte, den Einfluß von Talgzusatz bei Schmalz-Ölmischungen festzustellen. Auf S. 143 Zeile 5 von unten ist deshalb zu lesen: No. 56 anstatt No. 55 , ferner Zeile 3 von unten: No. 57 anstatt No. 56.

$K$. Alpers.

\section{Referate.}

\section{Mehle und Backwaren.}

A. Schleimer: Studien über das Gliadin, den alkobollöslichen Anteil des Weizenklebers. (Zeitschr. ges. Getreidewesen 1911, 3, 138-140, 194.) - Gliadin löst sich nicht etwa, wie man bisher annahm, am besten in Äthylalkohol von $70 \mathrm{Gew} . \%$, sondern vielmehr in solchem von $55 \mathrm{Gew} . \%$. Unter 30 und über 70 Gew. ${ }^{\circ} / 0$ Alkoholgehalt ist die Löslichkeit bedeutend herabgesetzt. Methylalkohol zeigt bei 60 Gew. $\%$ und Propylalkohol bei 40 Gew. $\%$ das beste Lösungsvermögen für Gliadin. Bei gleicher Konzentration der Alkohole steigt die Menge des gelösten Gliadins mit dem Molekulargewicht der Alkohole.

E. Dinslage.

R. Harcourt: Üb er gemälzten Weizen. (Zeitschr. ges. Getreidewesen 1911, 3, 191-194.) - Aus gemälztem Weizen hergestellte Mehle fallen um so dunkler aus, je weiter die Keimung vorangeschritten. Durch die Keimung wird der Gehalt an Amidstickstoff vermehrt, an Gluteninstickstoff vermindert, an alkohollöslichem (Gliadin-) Stickstoff nicht beeinfluBt; ferner vermindert sich der Gehalt an Stärke, während der an löslichen Kohlenhydraten zunimmt. Da lösliches Protein und lösliche Kohlenbydrate wesentliche Näbrstoffe der Hefe sind, erklärt sich die bessere Qualität des aus leicht gekeimtem Weizen hergestellten Gebäckes. Bei fortschreitender Keimung vermindert sich dagegen der Backwert des Weizens infolge weitgehender Veränderung der ursprünglichen Beschaffenheit des Klebers.

E. Dinslage.

M. P. Neumann, K. Mohs und H. Kalning: Uber den EinfluB der Lagerung und Trocknung auf die Beschaffenheit und Backfähigkeit des Weizenmehles. (Zeitschr. ges. Getreidewesen 1911, 3, 83-92.) - 